\begin{tabular}{|c|l|}
\hline Title & Identification of a $\beta$-glucosidase hydrolyzing tuberonic acid glucoside in rice (Oryza sativa L.) \\
\hline Author(s) & Wakuta, Shinji; Hamada, Shigeki; Ito, Hiroyuki; Matsuura, Hideyuki; Nabeta, Kensuke; Matsui, Hirokazu \\
\hline Citation & $\begin{array}{l}\text { Phytochemistry, 71(11-12), 1280-1288 } \\
\text { https://doi.org/10.1016/.phytochem.2010.04.025 }\end{array}$ \\
\hline Issue Date & $2010-08$ \\
\hline Doc URL & http://hdl.handle.net/2115/43767 \\
\hline Type & article(author version) \\
\hline File Information & Phy 71-11-12_1280-1288.pdf \\
\hline
\end{tabular}

Instructions for use 


\section{Identification of a $\beta$-glucosidase hydrolyzing tuberonic acid glucoside in rice (Oryza sativa $\mathrm{L}$.}

Shinji Wakuta ${ }^{\mathrm{a}}$, Shigeki Hamada ${ }^{\mathrm{a}, \mathrm{c}_{*}}$, Hiroyuki Ito ${ }^{\mathrm{b}}$, Hideyuki Matsuura ${ }^{\mathrm{a}}$, Kensuke Nabeta $^{\mathrm{a}}$, and Hirokazu Matsui ${ }^{\mathrm{a}}$

${ }^{a}$ Division of Applied Bioscience, Research Faculty of Agriculture, Hokkaido University, Sapporo 060-8589, Japan

${ }^{\mathrm{b}}$ Department of Materials Engineering, Akita National College of Technology, Akita 011-8511, Japan

${ }^{\mathrm{c}}$ National Institute of Crop Science, National Agriculture and Food Research Organization, Tsukuba, Ibaraki 305-8518, Japan

*Corresponding author. Tel./Fax: +8129838 8951

E-mail address: sty564@affrc.go.jp

Division of Applied Bioscience, Research Faculty of Agriculture

Hokkaido University, N-9, W-9, Kita-Ku

Sapporo 060-8589, Japan

Current address:

National Institute of Crop Science, National Agriculture and Food Research Organization, 2-1-18 Kannondai, Tsukuba, Ibaraki 305-8518, Japan 
1

2

Abbreviations: JA, jasmonic acid; TA, tuberonic acid; TAG, tuberonic acid glucoside; MS/MS, tandem mass spectrometry; UPLC, ultra-performance liquid chromatography; MRM, multiple reaction monitoring; pNPG, $p$-nitrophyenyl $\beta$-glucoside 


\begin{abstract}
Tuberonic acid (TA) and its glucoside (TAG) have been isolated from potato (Solanum tuberosum $L$.) leaflets and shown to exhibit tuber-inducing properties. These compounds were reported to be biosynthesized from jasmonic acid (JA) by hydroxylation and subsequent glycosylation, and to be contained in various plant species. Here we describe the in vivo hydrolytic activity of TAG in rice. In this study, the TA resulting from TAG was not converted into JA. Tuberonic acid glucoside (TAG)-hydrolyzing $\beta$-glucosidase, designated OsTAGG1, was purified from rice by six purification steps with 4300-fold purification. The purified enzyme migrated as a single band on native PAGE, but as two bands with molecular masses of 42 and $26 \mathrm{kDa}$ on SDS-PAGE. The results from N-terminal sequencing and peptide mass fingerprinting of both polypeptides suggested that the two bands were derived from a single polypeptide, which is a member of glycosyl hydrolase family 1 . In the native enzyme, the $K_{\mathrm{m}}$ and $V_{\max }$ values of TAG were $31.7 \mu \mathrm{M}$ and $14.7 \mu \mathrm{mol} / \mathrm{min} / \mathrm{mg}$, respectively. OsTAGG1 preferentially hydrolyzed TAG and methyl TAG. Here we report that OsTAGG1 is a specific $\beta$-glucosidase hydrolyzing TAG, which releases physiologically active TA.
\end{abstract}

Keywords: Oryza sativa L. cv. Kitaake; glycoprotein; $\beta$-glucosidase; tuberonic acid glucoside; tuberonic acid; enzyme purification 


\section{Introduction}

Jasmonic acid (JA), which is a member of oxylipins, is an essential compound involved in regulating responses to stresses such as insect herbivory and pathogenic attack in plants (McConn et al., 1997; Vijayan et al., 1998). In addition, JA is involved in plant growth and development, including root growth, fruit ripening, and pollen maturation (Staswick et al., 1992; Fan et al., 1998; Stintzi and Browse, 2000; Wasternack, 2007). Numerous genes have been previously identified that are inducible or down-regulated by JA (Sasaki et al., 2001; Wierstra and Kloppstech, 2000), suggesting that JA might be an important signaling molecule for plants in response to environmental changes and development.

JA is synthesized from the $\alpha$-linolenic acid released by lipase activity, followed by sequential reactions of peroxidation, dehydration, and cyclization to 12-oxo-phytodienoic acid (OPDA) (Song and Brash, 1991; Ishiguro et al., 2001; Schaller, 2001; Stenzel et al., 2003). OPDA is converted to JA by reduction and three $\beta$-oxidation steps (Vick and Zimmerman, 1984). JA is then metabolized by five basic reactions: (1) methylation at carbon 1 (Seo et al., 2001); (2) hydroxylation at carbon 11 or 12 (Helder et al., 1993; Yoshihara et al., 1989); (3) conjugation at carbon 1 with an amino acid and its analog (Sembdner et al., 1994); (4) reduction of the keto group at carbon 6 to form cucurbic acid (CA) (Dathe et al., 1991); and (5) degradation of the carboxylic acid side chain to form cis-jasmone (Koch et al., 1997). Hydroxylated JA is further modified by $O$-glucosylation or sulfation (Gidda et al., 2003; Seto et al., 2009). 12-OH-JA-Ile, 12-OH-JA-Phe (combination of (2) and (3) above), and 12-OH-CA 
(combination of (2) and (4) above) have also been identified (Guranowski et al., 2007). The existence of various JA analogs has made it difficult to determine the specific role of each compound in plant immunity and growth.

The genes involved in the JA biosynthetic pathway have been identified and well-studied. Analysis of Arabidopsis mutants showed that the genes DAD1, LOX2, $A O S$, and $O P R 3$ produce intermediates in the JA biosynthetic pathway (Bell et al., 1995; Stintzi and Browse, 2000; Ishiguro et al., 2001; Park et al., 2002). However, the enzymes that metabolize JA are still not known, although a few studies have been recently performed on corresponding mutants. JAR1 catalyzes the conversion of JA into JA-Ile, which promotes the interaction between $\mathrm{SCF}^{\mathrm{COI} 1}$ and $\mathrm{JAZ1}$; this leads to the ubiquitination of JAZ1 and its degradation by the 26S proteasome (Chini et al., 2007; Thines et al., 2007). JMT catalyzes the formation of MeJA from JA. This enzyme is essential for JA-regulated plant responses, and MeJA acts as a diffusible intercellular transducer (Seo et al., 2001). ST2a is able to sulfonate hydroxylated JA (Gidda et al., 2003). Thus, plants containing mutations in these enzymes should be used to provide information on the molecular function of compounds related to JA.

Tuberonic acid (TA), 12-hydroxyjasmonic acid (Figure 1), was isolated as a tuber-inducing compound from potato (Solanum tuberosum L.), and the glucoside of TA (TAG) was also found to be a tuberization compound (Koda et al., 1988; Yoshihara et al., 1989). In a previous study, it was suggested that TAG generated from JA transmits a signal from the damaged parts to the undamaged parts of the plant (Seto et al., 2009) and demonstrated that TA contributes to the down-regulation of the expression of JA 
biosynthetic genes, such as $L O X, A O S$, and $O P R$, which are JA-inducible. Furthermore, they demonstrated that some house-keeping genes, such as $R B C S, C A B 8$, and $T U B$, are also strongly down-regulated (Miersch et al., 2007). These data suggest the importance of TAG glucosidase as a regulator of TA levels according to environmental conditions. However, the TAG hydrolyzing enzyme has not been identified.

In this study, we describe the in vivo hydrolysis of TAG into TA in rice plants, purification and properties of an enzyme that catalyzes this hydrolysis, and identification of its gene. 


\section{Results}

\subsection{In vivo hydrolysis of TAG to TA}

We previously reported the synthesis of TAG from TA by glucosyltransferase in rice and tobacco plants (Seto et al., 2009). However, the mechanism of conversion of TAG into TA in plants is unclear (Figure 1). Since both TA and TAG are found in rice plants (Seto et al., 2009), we investigated the TAG hydrolyzing activity in rice during grain filling. Rice panicles and leaves were harvested, and then incubated in $\mathrm{H}_{2} \mathrm{O}$ (as a control) or 0.1 $\mathrm{mM}$ TAG- $d 5$ for $2 \mathrm{~h}$. The extracts from the treated materials were analyzed by ultra-performance liquid chromatography-tandem mass spectrometry (UPLC-MS/MS) as described in the Experimental section. Figure 2 shows the trace chromatograms in multiple reaction monitoring $(\mathrm{MRM})$ mode of standard TAG- $d 5$ detected by the daughter ion $\mathrm{m} / \mathrm{z}, 210.8$ derived from $\mathrm{m} / \mathrm{z}, 392.03$ and TA- $d 5$ detected by the daughter ion $m / z 96.6$ derived from $m / z$ 229.97. The retention times of TAG- $d 5$ and TA- $d 5$ were determined to be 1.95 and $2.14 \mathrm{~min}$, respectively (Figure $2 \mathrm{~A}$ and $2 \mathrm{~B}$ ). In the chromatograms of treated rice leaves, abundant TA- $d 5$ derived from hydrolyzing TAG- $d 5$ and the absorbed original TAG- $d 5$ were detected (Figure 2C). However, the corresponding peaks were not discernable from the background noise in the chromatograms of the control plants (Figure 2D). These data indicate the existence of a TAG hydrolyzing enzyme(s) that generates TA in rice plants.

\subsection{Purification of OsTAGG1 from immature rice seeds}


OsTAGG1 was purified from frozen rice panicles by ammonium sulfate fractionation and five chromatographic steps as shown in Table 1. Two peaks of activity for TAG glycosidase, designated OsTAGG1 and OsTAGG2, were detected by CM-Sepharose chromatography (data not shown), indicating that at least two isoforms of OsTAGG exist in rice panicles. OsTAGG1, which was detected as the major peak primarily eluted by CM-Sepharose chromatography, was further purified. After six purification steps, $0.065 \mathrm{mg}$ of OsTAGG1 was obtained from about $2 \mathrm{~kg}$ of rice panicles with 4300 -fold purification (Table 1). The purified enzyme migrated as a single band on native PAGE, but as two polypeptides with molecular masses of 42 and $26 \mathrm{kDa}$ on SDS-PAGE (Figure $3 \mathrm{~A}$ and $3 \mathrm{~B}$, left panel). The sum of the molecular masses of these polypeptides was roughly consistent with the molecular mass estimated by gel filtration chromatography (ca. $65 \mathrm{kDa}$, data not shown). Previous studies have shown that many $\beta$-glucosidases are glycoproteins (Hughes et al., 1992; Suzuki et al., 2006). Thus, the purified protein was also treated with a glycoprotein-staining reagent (Pro-Q Emerald 300 Gel Stain Kit), and the results indicated that both polypeptides constituting OsTAGG1 possessed sugar chains (Figure 3B, right panel), which was consistent with the binding of OsTAGG1 to ConA-Sepharose.

\subsection{Properties of OsTAGG1}

The enzymatic properties of the purified OsTAGG1 were investigated. OsTAGG1 was most active at around $\mathrm{pH} 4$ and $50^{\circ} \mathrm{C}$, and was fairly stable between $\mathrm{pH} 3.3-10.3$, since more than $90 \%$ of original activity was retained after incubation at $4{ }^{\circ} \mathrm{C}$ for $16 \mathrm{~h}$. When the enzyme was maintained for $15 \mathrm{~min}$ at various temperatures ranging from $30^{\circ} \mathrm{C}$ to 
$70^{\circ} \mathrm{C}$, the original activity was retained up to $50^{\circ} \mathrm{C}$ (Supplemental figure S1). When TAG was used as a substrate in the assay, the specific activity was $3.7 \mu \mathrm{mol} / \mathrm{min} / \mathrm{mg}$, and the Lineweaver-Burk plots of the reaction catalyzed by OsTAGG1 yielded $V_{\max }$ and $K_{\mathrm{m}}$ values of $14.7 \mu \mathrm{mol} / \mathrm{min} / \mathrm{mg}$ and $31.7 \mu \mathrm{M}$, respectively. The substrate specificity of the purified OsTAGG1 was studied in comparison with TAG and related compounds (Table 2). The relative activities for the hydrolysis of methyl TAG and jasmonyl-1- $\beta$-glucose were $80 \%$ and $51 \%$, respectively, of the value for TAG. OsTAGG1 efficiently hydrolyzed the synthetic substrate $p$-nitrophenyl $\beta$-D-glucoside (ca. 1.8-fold greater activity than that for TAG) but not $p$-nitrophenyl $\beta$-D-mannoside, $p$-nitrophenyl $\beta$-D-galactoside, $p$-nitrophenyl $\beta$-D-cellobioside, and $p$-nitrophenyl $\alpha$-D-glucoside. These data indicate that OsTAGG1 specifically recognizes the $\beta$-D-glucosidic linkage. Furthermore, when some natural glucosides, such as salicylic acid glucoside, linamarin, and zeatin- $O$-glucoside, were used as substrates, OsTAGG1 hydrolyzed only salicylic acid glucoside ( $25 \%$ of the value for TAG) but did not show any activity against linamarin and zeatin- $O$-glucoside. It was qualitatively confirmed that OsTAGG1 was able to hydrolyze to laminaribiose and cellotriose (data not shown).

\subsection{Identification of the OsTAGG1 gene and sequence comparison studies}

The purified OsTAGG1 showed two bands on SDS-PAGE with molecular masses of 42 and $26 \mathrm{kDa}$. The N-terminal amino acid sequences of both polypeptides were determined to be EPPPISRRSF and GLXNSYSTDA ( $\mathrm{X}$ indicates an unidentified amino acid), respectively. These sequences were found in the internal amino acid sequences of AK070962 (Os04g0474900) (Kikuchi et al., 2003), which was deposited as a cDNA of 
1853 bp containing a 1521-bp open reading frame encoding a 57-kDa polypeptide with 506 amino acid residues. The EPPPISRRSF sequence coincided with the N-terminal sequence generated by the cleavage of a signal sequence comprising the first 25 residues of the precursor protein as predicted by SignalP 3.0 (Bendtsen et al., 2004) for the AK070962 cDNA gene product. The tryptic digestion of both bands was analyzed by MALDI-TOF MS. The data obtained were entered into the MASCOT database and the same $\beta$-glucosidase showed a high score (data not shown). These results strongly suggest that both $42-$ and $26-\mathrm{kDa}$ polypeptides are encoded by a single gene (Os04g0474900). The molecular mass of the mature enzyme is predicted from the sequence to be ca. $55 \mathrm{kDa}$, but is inconsistent with the sum of the molecular masses of the two polypeptides $(68 \mathrm{kDa})$. This discrepancy may be explained by the addition of sugar chains. Indeed, there are five potential N-glycosylation sites at positions 118,225 , 357,367 , and 421 in the amino acid sequence of the precursor protein (Figure 4).

The predicted amino acid sequence of the OsTAGG1 precursor contained conserved amino acid residues among the GH1 members, such as Asn-Glu-Pro (residues 198-200) and Ile-Thr-Glu-Asn-Gly (residues 411-415). The glutamate residues positioned at 199 and 413 were predicted to be an acid-base catalyst and a catalytic nucleophile, respectively, based on previous studies (Withers et al., 1990; Wang et al., 1995). Residues involved in the binding of the sugar moiety of the substrate, which are highly conserved in all glycosyl hydrolase (GH) 1 members, were also found in the amino acid sequence of OsTAGG1; i.e., Gln-49, His-153, Asn-198, Glu-199, Glu-413, Trp-462, Glu-469, and Trp-470 (Figure 4) based on previous studies (Czjzek et al., 2000; Verdoucq et al., 2004). These results support the conclusion that OsTAGG1 is a 
member of the GH 1 family. High amino acid sequence identities were also found with rice Os4BGlu12 (87\%) (Opassiri et al., 2006), two hypothetical proteins from sorghum (Sorghum bicolor), accession numbers EES12355 (86\%) and EES12357 (78\%), and the putative rice $\beta$-glucosidases Os4BGlu9 (78\%), Os4BGlu10 (77\%), and Os4BGlu11 (79\%). Other enzymes exhibiting high amino acid sequence identities included the white clover cyanogenic $\beta$-glucosidase (ABV54716) (64\%) (Barrett et al., 1995), $\beta$-glucosidase D2 (Lotus japonicus) (ACD65510), which hydrolyzes hydroxynitrile glucosides (67\%) (Morant et al., 2008), an unnamed protein product from Vitis vinifera (CAO21978) (67\%), $\beta$-glucosidase (Rosa hybrid cultivar) (BAG13451) (65\%), isoflavone conjugate-hydrolyzing $\beta$-glucosidase from soybeans (Glycine max) (BAF34333) (62\%) (Suzuki et al., 2006), prunasin hydrolase (Prunus serotina) (AAF34650) (60\%) (Zhou et al., 2002), amygdalin hydrolase (P. serotina) (AAA93234) (58\%) (Zhou et at., 2002), all of which are known members of the GH1 family in plants (Figure 5) except for the uncharacterized enzymes. 


\section{Discussion}

The purified OsTAGG1 migrated as a single polypeptide on native PAGE, but as two polypeptides with molecular masses of $42 \mathrm{kDa}$ and $26 \mathrm{kDa}$ under denaturing conditions. Similar observations were reported in a previous study that the purified preparation of $\beta$-glucosidase from lodgepole pine xylem showed $28 \mathrm{kDa}$ and $24 \mathrm{kDa}$ proteins on SDS-PAGE, and it was concluded that this $\beta$-glucosidase should be a $55-\mathrm{kDa}$ monomeric enzyme (Dharmawardhana et al., 1995, 1999). The molecular mass of the native protein calculated by gel filtration was coincident with the sum of the two OsTAGG1 subunit bands on SDS-PAGE. Therefore, OsTAGG1 also appears to be a monomeric enzyme composed of two polypeptides produced by cleavage within a molecule in vivo or during purification similar to $\beta$-glucosidase from the lodgepole pine xylem.

TAG has an acidic group (carboxyl) in its aglycone moiety. Typical plant $\beta$-glucosidase substrates do not contain such an acidic aglycone moiety except glucosinolates, which are substrates for myrosinase. We performed primary sequence alignment between OsTAGG1 and myrosinase to predict the binding sites for negatively charged substrates (data not shown). The results showed that all amino acid residues involved in the interaction with the glucose moiety were conserved in OsTAGG1. However, two arginine sites, known as aglycone binding sites in a myrosinase (Burmeister et al., 1997), were not found in OsTAGG1. Furthermore, comparison with sorghum $\beta$-glucosidase, in which the crystal structure and aglycone binding sites have been analyzed, predicted almost no binding sites with an aglycone moiety, except S471 in OsTAGG1 according to $\mathrm{S} 462$ sorghum $\beta$-glucosidase. Therefore, we believe that other residues are involved 
in the interaction with the aglycone moiety of TAG.

TA is synthesized by hydroxylation of JA and then metabolized into TAG as evidenced by a time-dependent accumulation in a JA-dependent manner after wounding tomato leaves (Miersch et al., 2007). Furthermore, TA and TAG were not detected in a JA-deficient mutant (Miersch et al., 2007). A previous study showed that glucosyltransferase activity occurred towards TA in several plants and identified a TA glucosyltransferase in rice (Seto et al., 2009). The function of glycosylation is thought to be storage and/or transport of the bioactive aglycone in order to prepare for its release by $\beta$-glucosidase depending on a specific environmental cue such as wounding. However, the hydrolytic activity of TAG to generate TA has not been confirmed. In order to investigate the conversion of TAG to TA in vivo, TAG-d5 was synthesized. TA- $d 5$ was qualitatively detected in the rice plants fed with TAG- $d 5$, suggesting the existence of a specific $\beta$-glucosidase hydrolyzing TAG glucoside. We examined the possibility that TA was converted into JA in the same plant by feeding with TAG- $d 5$, but deuterium-labeled JA was undetectable. This result was completely consistent with a previous study in which labeled JA and $12-\mathrm{HSO}_{4}-\mathrm{JA}$ were undetectable after feeding with labeled TA (Gidda et al., 2003). These observations suggest that TAG has specific storage or transport forms for TA in vivo. Among JA metabolites, four glycoside conjugates have been reported apart from TAG: 11-hydroxyjasmonic acid glucoside (Matsuura et al., 2001), jasmonyl-1- $\beta$-glucose, jasmonyl-1- $\beta$-gentiobiose, and hydroxyjasmonyl-1- $\beta$-glucose (Swiatek et al., 2004). These compounds are classified as hydroxylated JA or JA after glucoside hydrolyzation, suggesting that glycoside conjugates may be creatively used to produce different bioactive hormones in vivo. 
While JA has been well studied as a plant hormone that protects against insect herbivory and pathogenic infections, the physiological functions and mechanisms of TA have been rarely reported. TA and TAG were reported to play a physiological role in inducing tubers (Yoshihara et al., 1989). Recent studies have indicated that TA is a physiologically active compound. For example, AtST2a, which is a hydroxyjasmonate sulfotransferase from Arabidopsis thaliana, was induced following TA treatment (Gidda et al., 2003). Wound-induced metabolic conversion of JA/MeJA into TA alters the expression pattern of genes including switching off JA signaling for the expression of a subset of genes (Miersch et al., 2007). In previous studies, TA and TAG were increased by mechanical wounding in rice and tobacco (Seto et al., 2009), supporting similar results found in tomato plants (Miersch et al., 2007). These results indicate the relationship between the wounding response in plants and TA and TAG accumulation. Furthermore, GH1 enzymes are involved in defense against herbivores and pathogens (Bjarnholt and Moller, 2008; Halkier and Gershenzon, 2006). Thus, OsTAGG1 may also be a specific $\beta$-glucosidase releasing TA, thereby providing a defense mechanism in plants. In future, analysis of the deletion variant will allow a better understanding of the function of OsTAGG1 and hydrolysis of TAG in plant defense responses.

Phytohormones such as abscisic acid, gibberellic acid, auxin, cytokinin, jasmonate, and brassinosteroids are metabolized to glycosylated conjugates (Sembdner et al., 1994). However, few studies have attempted to identify $\beta$-glucosidase involved in hydrolyzing these glycosylated phytohormones. Only TAG has been used as a substrate for two $\beta$-glucosidases, PYK10 (BGLU23) and BGLU18, which accumulate in constitutive and 
inducible ER bodies of A. thaliana (Ogasawara et al., 2009). A recent study showed that PYK10 and its close paralogs (BGLU21 and BGLU22) specifically hydrolyzed the natural substrate scopolin (Ahn et al., 2010). However, OsTAGG1 lies in a different cluster compared with PYK10 and BGLU18 (Figure 5), indicating that OsTAGG1 may have different functions compared with PYK10 and BGLU18 even TAG is a possible substrate for these enzymes. A previous study on the GH family 1 from rice showed that Os4bglu12 $\beta$-glucosidase also has high exoglucanase activity and is therefore predicted to play a role in cell wall metabolism (Opassiri et al., 2006). OsTAGG1 had the highest similarity to Os4bglu12 $\beta$-glucosidase and displayed hydrolytic activity towards laminaribiose and cellotriose (data not shown), indicating the possibility that OsTAGG1 is also involved in cell wall metabolism. Furthermore, the expression patterns of genes belonging to the rice $\mathrm{GH}$ family 1 showed that transcripts of OsTAGG1/Os4bglu13 accumulate in wounded tissues (Opassiri et al., 2006). These observations together with the remarkably high efficiency of TAG hydrolysis suggest that OsTAGG1 may be involved in the signal transduction of JA analogs via TAG to wound and/or disease damage.

\section{Conclusions}

In this study, the conversion of TAG to TA by $\beta$-glucosidase was demonstrated in rice, and rice TAG glucosidase (OsTAGG1) was purified. OsTAGG1 was shown to be a glycoprotein consisting of two polypeptides derived from a single gene 
1

2

(Os04g0474900) (AK070962). The enzyme showed high specificity for TAG compared to other glucosidic plant hormones. In order to investigate in more detail the function of OsTAGG1 in vivo, overexpressed and/or deficient mutants must be analyzed. 


\section{Experimental}

\subsection{Plant materials and chemicals}

Plants (O. sativa L. cv. Kitaake) to be used for enzyme purification and the in vivo TAG hydrolyzing assay were grown in an experimental field at Hokkaido University, Sapporo, Japan, and in a growth chamber, respectively. The chamber conditions were set at $30^{\circ} \mathrm{C}$, $60 \%$ moisture, and $530 \mu \mathrm{mol} / \mathrm{m}^{2} / \mathrm{s}$ illumination under a 10.5 -h light period. TA, TAG, deuterium-labeled TA (TA- $d 5)$, deuterium-labeled TAG (TAG- $d 5)$, and jasmonyl-1- $\beta$-glucoside were prepared as described previously (Matsuura et al., 2000). Linamarin, zeatin- $O$-glucoside, salicylic acid glucoside, and pNPG were obtained from Sigma Chemicals (St. Louis, MO). D-glucose- ${ }^{13} \mathrm{C}_{6}$ was purchased from Taiyo Nissan (Tokyo, Japan).

\subsection{Purification of TAG glucosidase (OsTAGG)}

Plant materials from rice, including the panicles, were harvested during grain filling (ca. $2 \mathrm{~kg}$, fr. wt). These materials were frozen in liquid nitrogen and powdered using a blender (Nihonseiki Kaisha Ltd., Tokyo, Japan). The powdered materials were suspended in 41 extraction buffer $(20 \mathrm{mM}$ phosphate buffer, $\mathrm{pH} 7.0$, containing $1 \mathrm{mM}$ DTT and $1 \mathrm{mM}$ phenylmethylsulfonyl fluoride) and homogenized using a Polytron (Kinematica $\mathrm{GmbH}$, Switzerland). A crude extract was obtained by filtering through two layers of gauze and centrifugation at $12,000 \times g$ for $20 \mathrm{~min}$ at $4^{\circ} \mathrm{C}$. OsTAGG protein was purified from the crude extract by the following procedure: 
The protein fraction that was precipitated in $40 \%-80 \%$ saturation of $\left(\mathrm{NH}_{4}\right)_{2} \mathrm{SO}_{4}$ was collected by centrifugation at $12,000 \times g$ for $20 \mathrm{~min}$ at $4^{\circ} \mathrm{C}$. The pellet was dissolved in buffer A (Macllvaine, $\mathrm{pH}$ 5.0) and then dialyzed against buffer A. The dialyzed sample was loaded on a CM-Sepharose Fast Flow column $(30 \times 280 \mathrm{~mm}$, GE Healthcare Bio-Sciences, Piscataway, NJ) equilibrated with buffer A. After the column was washed with buffer A, proteins were eluted with a linear gradient of $0-0.5 \mathrm{M} \mathrm{NaCl}$ in buffer $\mathrm{A}$. The active fractions were pooled and dialyzed against buffer B $(20 \mathrm{mM}$ phosphate buffer, $\mathrm{pH} 7.0$, containing $0.5 \mathrm{M} \mathrm{NaCl}$ ). The retained fraction was applied to a Con A-Sepharose 4B column $(16 \times 100 \mathrm{~mm}$, GE Healthcare $)$ equilibrated with buffer B, and proteins were eluted with $0.5 \mathrm{M}$ mannose. The active fractions were pooled, concentrated, and then fractionated onto a HiLoad 16/60 Superdex 200 pg column $(16 \times$ $600 \mathrm{~mm}$, GE Healthcare) equilibrated with buffer A containing $0.2 \mathrm{M} \mathrm{NaCl}$. Proteins were eluted at a flow rate of $0.8 \mathrm{ml} / \mathrm{min}$ using an ÄKTA system (GE Healthcare). The active fractions were pooled and dialyzed against buffer $\mathrm{A}$ and then fractionated on Resource S (1 ml; GE Healthcare) equilibrated with the same buffer. The protein was eluted with a linear gradient of $0-0.2 \mathrm{M} \mathrm{NaCl}$ in buffer A. Finally, the active fractions were pooled and dialyzed against buffer $\mathrm{C}(10 \mathrm{mM}$ phosphate buffer, $\mathrm{pH} 7.0)$ and resolved on hydroxyapatite (25 ml; Seikagaku Kougyou, Tokyo, Japan) equilibrated with buffer $\mathrm{C}$. The enzyme was eluted with a linear gradient of 10-200 mM phosphate buffer. The purified OsTAGG was pooled, concentrated using a Vivaspin centrifugal filtration device with a molecular weight cut-off (Vivascience, Hanover, Germany), and then stored at $-20^{\circ} \mathrm{C}$ after adding glycerol (final concentration $40 \%$ ).

\subsection{UPLC and MS conditions}


The analytical procedures for TA and TAG were according to a previous study (Matsuura et al., 2009). In the analysis for glucose, UPLC separation was performed on a Waters Acquity ethylene-bridged $(\mathrm{BEH}) \mathrm{C}_{18}$ column $(1.7 \mu \mathrm{m}, 2.1 \times 100 \mathrm{~mm})$ at $38^{\circ} \mathrm{C}$. The analytes were eluted from the column with a solvent of $\mathrm{MeOH}-\mathrm{H}_{2} \mathrm{O}(1: 4, \mathrm{v} / \mathrm{v}$; solvent A) at an isocratic mode. MS optimization experiments and all quantifications were performed in the MS scan and MRM modes, respectively. The tune page parameters and conditions for each MRM transition were optimized by infusing the 100 ppm standard solution into the mass spectrometer. To ensure that the tune page parameters were compatible with the UPLC flow during tuning, a UPLC flow of 0.3 $\mathrm{ml} / \mathrm{min}$ for solvent A was introduced into the mass spectrometer at the same time by utilizing a $T$ unit (Upchurch Scientific, Oak Harbor, WA, USA). To collect the MRM data during the UPLC experiments, the capillary voltage was $3.0 \mathrm{kV}$, source temperature was $120^{\circ} \mathrm{C}$, desolation temperature was $350^{\circ} \mathrm{C}$, desolation gas flow was $800 \mathrm{l} / \mathrm{h}$, and cone gas flow was $50 \mathrm{l} / \mathrm{h}$. During each UPLC injection, the mass spectrometer was set to collect data in the MRM mode with electrospray ionization in the negative ion mode. The MRM transitions for the tested compounds were as follows: glucose $\left\{[\mathrm{M}-\mathrm{H}]^{-}\right.$: 178.80, transition ion $(\mathrm{m} / \mathrm{z}): 88.4$, cone voltage: $25.5 \mathrm{~V}$, collision energy: $9.00 \mathrm{eV}\}$ and D-glucose- ${ }^{13} \mathrm{C}_{6}\left\{[\mathrm{M}-\mathrm{H}]^{-}:\right.$: 184.87, transition ion $(\mathrm{m} / \mathrm{z}): 91.4$, cone voltage: $25.0 \mathrm{~V}$, collision energy: $10.00 \mathrm{eV}$ \}.

\subsection{Enzyme assays}

The reaction mixture (final volume, $100 \mu \mathrm{l}$ ) consisting of $10 \mu \mathrm{M}$ TAG and $20 \mathrm{mM}$ 
$\mathrm{NaOAc}$ buffer ( $\mathrm{pH} 4.0$ ) was preincubated at $37^{\circ} \mathrm{C}$ for $3 \mathrm{~min}$. The reaction was initiated by adding the enzyme, incubating at $37^{\circ} \mathrm{C}$ for $10 \mathrm{~min}$, and then terminating the reaction by adding $90 \mu \mathrm{l}$ of $100 \% \mathrm{MeOH}$. After adding $2.5 \mu \mathrm{M}$ (final concentration) TA- $d 5$ as an internal standard, the mixture was analyzed using the UPLC-MS/MS system. The amount of the reaction product was calculated by comparing the peak areas of TA- $d 5$. When the optimum $\mathrm{pH}$ was determined, McIlvaine's buffer ( $\mathrm{pH}$ 3.0-7.0) was used as a reaction buffer instead of NaOAc. To determine the optimum temperature, the purified enzymes were reacted at different temperatures $\left(20^{\circ} \mathrm{C}-65^{\circ} \mathrm{C}\right)$ in the abovementioned standard conditions. After $\mathrm{pH}$ stability was analyzed, the purified proteins were treated for $16 \mathrm{~h}$ at different $\mathrm{pHs}$ in Britton-Robinson's buffer ( $\mathrm{pH} 2.3-11.3)$. Then the treated protein was diluted 10-fold with $0.2 \mathrm{M} \mathrm{NaOAc}$ buffer $(\mathrm{pH} 4.0$ ) for reaction. The purified proteins were heat-treated at different temperatures $\left(30^{\circ} \mathrm{C}-70^{\circ} \mathrm{C}\right)$ for $15 \mathrm{~min}$, and then immediately cooled on ice to analyze the residual activity. The kinetic parameters were derived from the Lineweaver-Burk plots. The effect of substrate concentration on reaction velocity was examined at various concentrations $(7,10,15,25$, 40 , and $70 \mu \mathrm{M}$ ) of TAG. The enzyme activity showed a constant velocity for $10 \mathrm{~min}$ even in the lowest TAG concentration $(7 \mu \mathrm{M})$. For each substrate specificity analysis, the enzyme was incubated with $50 \mu \mathrm{M}$ of each glycosidic compound (Table 2) and then the released glucose, mannose, or galactose was quantified with $2.5 \mu \mathrm{M}$ glucose- ${ }^{13} \mathrm{C}_{6}$ as an internal standard using the UPLC-MS/MS system. These standard curves (glucose, mannose, or galactose) were measured by the methods described in the section on the UPLC and MS conditions and showed linearity for $0-50 \mu \mathrm{M}$ concentrations.

\subsection{Determination of molecular mass}


The molecular mass was determined by gel filtration on a Superose 12 10/300 GL (GE Healthcare $)$ column and by SDS-PAGE. The Superose column $(10 \times 300 \mathrm{~mm})$ was equilibrated with buffer A containing $0.2 \mathrm{M} \mathrm{NaCl}$ and then the purified enzyme $(6 \mu \mathrm{g})$ was run. The molecular mass calibration was performed by eluting standard proteins in the range of $1.35-670 \mathrm{kDa}$ (Bio-Rad, Hercules, CA). SDS-PAGE was performed with a $15 \%(w / v)$ acrylamide separation gel. Protein mass markers in the range of 14.4-97.4 kDa (Bio-Rad) were used. Protein bands were visualized by staining with Coomassie brilliant blue R-250 (CBB).

\subsection{MALDI-TOF MS and N-terminal amino acid sequence analyses}

About $1 \mu \mathrm{g}$ of purified enzyme was resolved by electrophoresis on a $15 \%$ SDS-PAGE gel (Figure 3B). After staining with CBB, the stained gel band was excised from the gel. The gel pieces were thoroughly destained with $25 \mathrm{mM} \mathrm{NH}_{4} \mathrm{HCO}_{3}$ in $50 \%$ acetonitrile and dried under vacuum. A total of $20 \mu \mathrm{l}$ of $10 \mu \mathrm{g} / \mathrm{ml}$ Trypsin Gold solution (mass spectrometry grade; Promega, Madison, WI) was added, and the tube was placed on ice for $30 \mathrm{~min}$ and then placed at $37^{\circ} \mathrm{C}$ for $12 \mathrm{~h}$ for tryptic digestion. The digested peptides were eluted twice with $100 \mu \mathrm{l}$ of $75 \%$ acetonitrile containing $0.1 \%$ TFA. After concentration, the sample was desalted using a ZipTip C18 tip (Millipore, Billerica, MA) and analyzed on a Voyager MALDI-TOF mass spectrometer (Applied Biosystems, Foster City, CA). 2,5-Dihydroxy benzoic acid $(10 \mathrm{mg} / \mathrm{ml})$ was used as the matrix, and the laser power was set at 2500 in refract mode. The MASCOT database (http://www.matrixscience.com/) was searched to identify the proteins found. The 
$\mathrm{N}$-terminal amino acid sequence was determined using a protein sequencer (Procise 491; Applied Biosystems) after the peptides of the purified enzyme were transferred onto a polyvinylidene difluoride membrane from SDS-PAGE using a Trans-Blot Semi-Dry Transfer Cell (Bio-Rad).

\section{Acknowledgments}

This work was supported by Grants-in-Aid for Young Scientists (B) (20780069) to SH from the Ministry of Education, Science, Sports, and Culture, Japan. 


\section{References}

Ahn, Y.O., Shimizu, B., Sakata, K., Gantulga, D., Zhou, Z., Bevan, D.R., Esen, A., 2010. Scopolin-hydrolyzing $\beta$-glucosidases in roots of Arabidopsis. Plant and Cell Physiol. 51, $132-143$.

Barrett, T., Suresh, C.G., Tolley, S.P., Dodson, E.J., Hughes, M.A. 1995. The crystal structure of a cyanogenic $\beta$-glucosidase from white clover, a family 1 glycosyl hydrolase. Structure. 15, 951-960.

Bell, E., Creelman, R.A., Mullet, J.E., 1995. A chloroplast lipoxygenase is required for wound-induced jasmonic acid accumulation in Arabidopsis. Proc. Natl. Acad. Sci. USA $92,8675-8679$.

Bendtsen, J.D., Nielsen, H., von Heijne, G., Brunak, S., 2004. Improved prediction of signal peptides: SignalP 3.0. J. Mol. Biol. 340, 783-795.

Bjarnholt, N., Moller, B.L., 2008. Hydroxynitrile glucosides. Phytochemistry 69, 1947-1961.

Burmeister, W.P., Cottaz, S., Driguez, H., Iori, R., Palmieri, S., Henrissat, B., 1997. The crystal structures of Sinapis alba myrosinase and a covalent glycosyl-enzyme intermediate provide insights into the substrate recognition and active-site machinery of an $S$-glycosidase. Structure 15, 663-675. 
Chini, A., Fonseca, S., Fernandez, G., Adie, B., Chico, J.M., Lorenzo, O., Garcia-Casado, G., Lopez-Vidriero, I., Lozano, F.M., Ponce, M.R., Micol, J.L., Solano, R., 2007. The JAZ family of repressors is the missing link in jasmonate signaling. Nature 448, 666-671.

Czjzek, M., Cicek, M., Zamboni, V., Bevan, D. R., Henrissat, B., Esen, A. 2000. The mechanism of substrate (aglycone) specificity in $\beta$-glucosidases is revealed by crystal structures of mutant maize $\beta$-glucosidases-DIMBOA, -DIMBOAGlc, and -dhurrin complexes. Proc. Natl. Acad. Sci. USA 97, 13555-13560.

Dathe, W., Schindler, C., Schneider, G., Schmidt, J., Porzel, A., Jensen, E., Yamaguchi, I., 1991. Cucurbic acid and its 6,7-stereoisomers. Phytochemistry 30, 1909-1914.

Dharmawardhana, D.P., Ellis, B.E., Carlson, J.E., 1995. A $\beta$-glucosidase from lodgepole pine xylem specific for the lignin precursor coniferin. Plant Physiol. 107, 331-339.

Dharmawardhana, D.P., Ellis, B.E., Carlson, J.E., 1999. cDNA cloning and heterologous expression of coniferin $\beta$-glucosidase. Plant Mol. Biol. 40, 365-372.

Fan, X., Mattheis, J.P., Fellman, J.K., 1998. A role for jasmonates in climacteric fruit ripening. Planta 204, 444-449.

Gidda, S.K., Miersch, O., Levitin, A., Schmidt, J., Wasternacks, C., Varin, L., 2003. 
Biochemical and molecular characterization of a hydroxyjasmonate sulfotransferase from Arabidopsis thaliana. J. Biol. Chem. 278, 17895-17900.

Guranowski, A., Miersch, O., Staswick, P.E., Suza, W., Wasternack, C., 2007. Substrate specificity and products of side-reaction catalyzed by jasmonate:amino acid synthetase (JAR1). FEBS Lett. 581, 815-820.

Halkier, B.A., Gershenzon, J., 2006. Biology and biochemistry of glucosinolates. Annu. Rev. Plant Biol. 57, 303-333.

Helder, H., Miersch, O., Vreugdenhil, D., Sembdner, G., 1993. Occurrence of hydroxylated jasmonic acids in leaflets of Solanum demissum plants grown under longand short-day conditions. Physiol. Plant. 88, 647-653.

Hughes, M.A., Brown, K., Pancoro, A., Murray, B.S. Oxtoby, E., Hughes, J., 1992. A molecular and biochemical analysis of the structure of the cyanogenic $\beta$-glucosidase (Linamarase) from cassava (Manihot esculenta Crantz). Arch. Biochem. Biophys. 295, 273-279.

Ishiguro, S., Kawai-Oda, A., Ueda, J., Nishida, I., Okada, K., 2001. The DEFECTIVE IN ANTHER DEHISCENCE1 gene encodes a novel phospholipase A1 catalyzing the initial step of jasmonic acid biosynthesis, which synchronizes pollen maturation, anther dehiscence, and flower opening in Arabidopsis. Plant Cell 13, 2191-2209. 
Kikuchi, S., Satoh, K., Nagata, T., Kawagashira, N., Doi, K., Kishimoto, N., Yazaki, J., Ishikawa, M., Yamada, H., Ooka, H., Hotta, I., Kojima, K., Namiki, T., Ohneda, E., Yahagi, W., Suzuki, K., Li, C.J., Ohtsuki, K., Shishiki, T., Otomo, Y., Murakami, K., Iida, Y., Sugano, S., Fujimura, T., Suzuki, Y., Tsunoda, Y., Kurosaki, T., Kodama, T., Masuda, H., Kobayashi, M., Xie, Q., Lu, M., Narikawa, R., Sugiyama, A., Mizuno, K., Yokomizo, S., Niikura, J., Ikeda, R., Ishibiki, J., Kawamata, M., Yoshimura, A., Miura, J., Kusumegi, T., Oka, M., Ryu, R., Ueda, M., Matsubara, K., Kawai, J., Carninci, P., Adachi, J., Aizawa, K., Arakawa, T., Fukuda, S., Hara, A., Hashizume, W., Hayatsu, N., Imotani, K., Ishii, Y., Itoh, M., Kagawa, I., Kondo, S., Konno, H., Miyazaki, A., Osato, N., Ota, Y., Saito, R., Sasaki, D., Sato, K., Shibata, K., Shinagawa, A., Shiraki, T., Yoshino, M., Hayashizaki, Y., Yasunishi, A., 2003. Collection, mapping, and annotation of over 28,000 cDNA clones from japonica rice. Science 301, 376-379.

Koch, T., Bandemer, K., Boland, W., 1997. Biosynthesis of cis-jasmone: A pathway for the inactivation and the disposal of the plant stress hormone jasmonic acid to the gas phase? Helvetica Chimica Acta 80, 838-850.

Koda, Y., Omer, E.A., Yoshihara, T., Shibata, H., Sakamura, S., Okazawa, Y., 1988. Isolation of a specific potato tuber-inducing substance from potato leaves. Plant Cell Physiol. 29, 1047-1051.

Matsuura, H., Ohmori, F., Kobayashi, M., Sakurai, A., Yoshihara, T., 2000. Qualitative and quantitative analysis of endogenous jasmonoids in potato plant (Solanum tuberosum L.). Biosci. Biotechnol. Biochem. 64, 2380-2387. 
Matsuura, H., Ohkubo, Y., Yoshihara, T., 2001. Occurrence of 11-hydroxyjasmonic acid glucoside in leaflets of potato plants (Solanum tuberosum L.). Biosci. Biotechnol. Biochem. 65, 378-382.

Matsuura, H., Aoi, A., Satou, C., Nakaya, M., Masuta, C., Nabeta, K., 2009. Simultaneous UPLC MS/MS analysis of endogenous jasmonic acid, salicylic acid, and their related compounds. Plant Growth Regul. 57, 293-301.

McConn, M., Creelman, R.A., Bell, E., Mullet, J.E., Browse, J., 1997. Jasmonate is essential for insect defense in Arabidopsis. Proc. Natl. Acad. Sci. USA 94, 5473-5477.

Miersch, O., Neumerkel, J., Dippe, M., Stenzel, I., Wasternack, C., 2007. Hydroxylated jasmonates are commonly occurring metabolites of jasmonic acid and contribute to a partial switch-off in jasmonate signaling. New Phytologist 177, 114-127.

Morant, A.V., Bjarnholt, N., Kragh, M.E., Kjærgaard, C.H., Jørgensen K., Paquette, S.M., Piotrowski, M., Imberty, A., Olsen, C.E., Møller, B.L., Bak, S., 2008. The $\beta$-glucosidases responsible for bioactivation of hydroxynitrile glucosides in Lotus japonicus. Plant Physiol. 147, 1072-1091.

Ogasawara, K., Yamada, K., Christeller, J.T., Kondo, M., Hatsugai, N., Hara-Nishimura, I., Nishimura, M., 2009. Constitutive and inducible ER bodies of Arabidopsis thaliana accumulate distinct $\beta$-glucosidases. Plant Cell Physiol. 50, 480-488. 
Opassiri, R., Pomthong, B., Onkoksoong, T., Akiyama, T., Esen, A., Ketudat Cairns, J.R., 2006. Analysis of rice glycosyl hydrolase family I and expression of Os4bglu12 $\beta$-glucosidases. BMC Plant Biology, 6:33.

Park, J.H., Halitschke, R., Kim, H.B., Baldwin, I.T., Feldmann, K.A., Feyereisen, R., 2002. A knock-out mutation in allene oxide synthase results in male sterility and defective wound signal transduction in Arabidopsis due to a block in jasmonic acid biosynthesis. Plant J. 31, 1-12.

Qian, Z.G., Zhao, Z.J., Xu, Y., Qian, X., Zhong, J.J., 2004. Novel chemical synthesized hydroxyl-containing jasmonate as powerful inducing signals for plant secondary metabolism. Biotechnol. Bioeng. 86, 809-816.

Sasaki, Y., Asamizu, E., Shibata, D., Nakamura, Y., Kaneko, T., Awai, K., Amagai, M., Kuwata, C., Tsugane, T., Masuda, T., Shimada, H., Takamiya, K., Ohta, H., Tabata, S., 2001. Monitoring of methyl jasmonate-responsive genes in Arabidopsis by cDNA macroarray: Self-activation of jasmonic acid biosynthesis and crosstalk with other phytohormone signaling pathways. DNA Res. 8, 153-161.

Schaller, F., 2001. Enzymes of the biosynthesis of octadecanoid-derived signalling molecules. J. Exp. Bot. 52, 11-23.

Sembdner, G., Atzorn, R., Schneider, G., 1994. Plant hormone conjugation. Plant Mol. 
Biol. 26, 1459-1481.

Seo, H.S., Song, J.T., Cheong, J.J., Lee, Y.H., Lee, Y.W., Hwang, I., Lee, J.S., Choi, Y.D., 2001. Jasmonic acid carboxy methyltransferase: A key enzyme for jasmonate-regulated plant responses. Proc. Natl. Acad. Sci. USA 98, 4788-4793.

Seto, Y., Hamada, S., Matsuura, H., Matsushige, M., Satou, C., Takahashi, K., Masuta, C., Ito, H., Matsui, H., Nabeta, K., 2009. Purification and cDNA cloning of a wound inducible glucosyltransferase active toward 12-hydroxy jasmonic acid. Phytochemistry $70,370-379$

Song, W.C., Brash, A.R., 1991. Purification of an allene oxide synthase and identification of the enzyme as a cytochrome P-450. Science 253, 781-784.

Staswick, P.E., Su, W., Howell, S.H., 1992. Methyl jasmonate inhibition of root growth and induction of a leaf protein are decreased in an Arabidopsis thaliana mutant. Proc. Natl. Acad. Sci. USA 89, 6837-6840.

Stenzel, I., Hause, B., Miersch, O., Kurz, T., Maucher, H., Weichert, H., Ziegler, J., Feussner, I., Wasternack, C., 2003. Jasmonate biosynthesis and the allene oxide cyclase family of Arabidopsis thaliana. Plant Mol. Biol. 51, 895-911.

Stintzi, A., Browse, J., 2000. The Arabidopsis male-sterile mutant, opr3, lacks the 12-oxophytodienoic acid reductase required for jasmonate synthesis. Proc. Natl. Acad. 
Sci. USA 97 10625-10630.

Suzuki, H., Takahashi, S., Watanabe, R., Fukushima, Y., Fujita, N., Noguchi, A., Yokoyama, R., Nishitani, K., Nishino, T., Nakayama, T., 2006. An isoflavone conjugate-hydrolyzing $\beta$-glucosidase from the roots of soybean (Glycine max) seedlings. J. Biol. Chem. 281, 30251-30259.

Swiatek, A., Dongen, W.V., Esmans, E.L., Onckelen, H.V., 2004. Metabolic fate of jasmonates in Tobacco Bright Yellow-2 cells. Plant Physiol. 135, 161-172.

Thines, B., Katsir, L., Melotto, M., Niu, Y., Mandaokar, A., Liu, G., Nomura, K., He, S.Y., Howe, G.A., Browse, J., 2007. JAZ repressor proteins are targets of the SCFCOI1 complex during jasmonate signaling. Nature 448, 661-665.

Verdoucq, L., Morinière, J., Bevan, D.R., Esen, A., Vasella, A., Henrissat, B., Czjzek, M., 2004. Structural determinants of substrate specificity in family $1 \beta$-glucosidases. J. Biol. Chem. 279, 31796-31803.

Vick, B.A., Zimmerman, D.C., 1984. Biosynthesis of jasmonic acid by several plant species. Plant Physiol. 75, 458-461.

Vijayan, P., Shockey, J., Lévesque, C.A., Cook, R.J., Browse, J., 1998. A role for jasmonate in pathogen defense of Arabidopsis. Proc. Natl. Acad. Sci. USA 95, 7209-7214. 


\begin{abstract}
Wang, Q., Trimbur, D., Graham, R., Warren, R.A.J., Withers, S.G., 1995. Identification of the acid/base catalyst in Agrobacterium faecalis $\beta$-glucosidase by kinetic analysis of mutants. Biochemistry 34, 14554-14562.
\end{abstract}

\begin{abstract}
Wasternack, C., 2007. Jasmonates: An update on biosynthesis, signal transduction and action in plant stress response, growth and development. Annals of Botany 100, 681-697.
\end{abstract}

Wierstra, I., Kloppstech, K., 2000. Differential effects of methyl jasmonate on the expression of the early light-inducible proteins and other light-regulated genes in barley. Plant Physiol. 124, 833-844.

Withers, S.G., Warren, R.A.J., Street, I.P., Rupitz, K., Kempton, J.B., Aebersold, R., 1990. Unequivocal demonstration of the involvement of a glutamate residue as a nucleophile in the mechanism of a "Retaining" glycosidase. J. Am. Chem. Soc. 112, 5887-5889.

Yoshihara, T., Omer, E.A., Koshino, H., Sakamura, S., Kikuta, Y., Koda, Y., 1989. Structure of a tuber-inducing stimulus from potato leaves (Solanum tuberosum L.). Agric. Biol. Chem. 53, 2835-2837.

Zhou, J., Hartmann, S., Shepherd, B.K., Poulton, J.E., 2002. Investigation of the microheterogeneity and aglycone specificity-conferring residues of black cherry 
1

2

3

4

5

6

7

8

9

10

11

12

13

14

15

16

17

18

19

20

21

22

23

24

25

26

27

28

29

30

31

32

33

34

35

36

37

38

39

40

41

42

43

44

45

46

47

48

49

50

51

52

53

54

55

56

57

58

59

60

61

62

63

64

65

prunasin hydrolases. Plant Physiol. 129, 1252-1264. 


\section{Figure legends}

Fig. 1. Metabolism of jasmonic acid focusing on TA and TAG. The narrow arrows indicate TAG biosynthesis. JA is metabolized to TA by hydroxylation at the $\mathrm{C}-12$ position and then glucosylated to produce TAG. Bold arrows show the reverse pathway generating TA from TAG as described in this study.

Fig. 2. UPLC-MS/MS MRM chromatograms for TAG- $d 5$ and TA- $d 5$. The trace chromatogram in the MRM mode shows the ion peaks $m / z 392.03 / 210.8$ for TAG- $d 5$ and 229.97/96.6 for TA- $d 5$. (A) and (B), Synthesized TAG- $d 5$ and TA- $d 5$ as standards; (C) Crude extraction of rice leaves treated with $0.1 \mathrm{mM}$ TAG- $d 5$; (D) Crude extraction of control plant. The open and closed arrows indicate the detection peaks for TAG- $d 5$ and TA- $d 5$, respectively.

Fig. 3. SDS-PAGE and native PAGE of purified OsTAGG1. (A) OsTAGG1 was loaded on $10 \%$ polyacrylamide and CBB-stained native PAGE gels. (B) Total protein was stained with $\mathrm{CBB}$ (left panel) and Pro-Q emerald for glycoprotein detection (right panel). Lane $\mathrm{M}$ was loaded with standards: myosin (200 kDa), $\beta$-galactosidase (116 $\mathrm{kDa})$, phosphorylase $b(97 \mathrm{kDa})$, bovine serum albumin (66 kDa), ovalbumin (45 kDa), bovine carbonic anhydrase $(31 \mathrm{kDa})$, and soybean trypsin inhibitor $(22 \mathrm{kDa})$. The arrow shows the 42- and 26-kDa polypeptides that match with $\beta$-glucosidase (AK070962) according to N-terminal amino acid and MALDI-TOF MS analyses.

Fig. 4. Nucleotide and deduced amino acid sequences of the cDNA encoding OsTAGG1. 
The circles and closed triangles denote the catalytic and sugar-binding site residues that are highly conserved in glycoside hydrolase family 1 , respectively. The open triangle denotes the serine that is identical to the corresponding aglycone binding site in sorghum dhurrinase-1. The sequences corresponding to the $\mathrm{N}$-terminal sequences of large and small subunits of purified OsTAGG1 on SDS-PAGE are underlined (see also Figure 3B). The circled highlighted residues indicate predictive N-glycosylation sites.

Fig. 5. A phylogenetic tree of the amino acid sequences of the glycoside hydrolase family 1 from various plants. The tree was constructed using ClustalX (version 2.0.11) (neighbor-joining method) and visualized using TreeView. Numbers indicate bootstrap values greater than 500. The position of OsTAGG1 is boxed. Proteins used for alignment without OsTAGG1 are as follows: hypothetical protein (Sorghum bicolor), EES12355; hypothetical protein (S. bicolor), EES12357; unnamed protein product (Vitis vinifera), CAO21978; $\beta$-glucosidase (Rosa hybrid cultivar), BAG13451; dalcochinin 8'-O- $\beta$-glucosidase (Dalbergia cochinchinensis), AAF04007; prunasin hydrolase (Prunus serotina), AAF34650; amygdalin hydrolase (P. serotina), AAA93234; cynogenic $\quad \beta$-glucosidase (Trifolium repens), ABV54716; $\quad$ isoflavone conjugate-specific- $\beta$-glucosidase (Glycine max), BAF34333; furostanol glycoside 26-O- $\beta$-glucosidase (Costus speciosus), BAA11831; $\beta$-glucosidase D2 (Lotus japonicus), ACD65510; coniferin $\beta$-glucosidase (Pinus contorta), AAC69619; linamarase (Manihot esculenta), AAB22162; $\beta$-primeverosidase (Camellia sinensis), BAC78656; $\beta$-mannosidase (Lycopersicon esculentum), AAL37714; strictosidine $\beta$-glucosidase (Catharanthus roseus), AAF28800; zeatin-O-glucoside degrading 
$\beta$-glucosidase (Brassica napus), CAA57913; $\beta$-glucosidase (Zea mays), AAB03266; raucaffricine-O- $\beta$-glucosidase (Rauwolfia serpentina), AAF03675; vicianin hydrolase (Vicia sativa), ABD03937; furcatin hydrolase (Viburnum furcatum), BAD14925; cardenolide 16-O-glucohydrolase (Digitalis lanata), CAB38854; dhurrinase (Sorghum bicolor), AAC49177; myrosinase (Sinapis alba), CAA42534; BGLU18 (Arabidopsis thaliana), AAF22295; PYK10 (A. thaliana), NP_187537. Other rice $\beta$-glycosidases similar to OsTAGG1 are shown as Os4bglu9 (B7ECS8), Os4bglu10 (Q7F9K4), Os4bglu11 (Q7XKV5), and Os4bglu12 (Q7XKV4). 


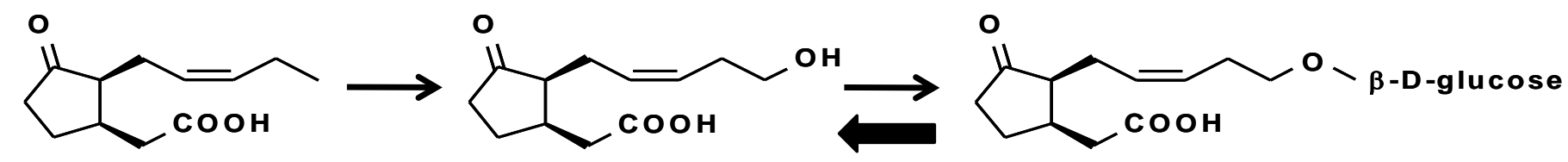

(+)-7-iso jasmonic acid

(JA)
Tuberonic acid

(TA)
Tuberonic acid $\beta$-D-glucose

(TAG)

Figure 1. Wakuta et al. 
A

B
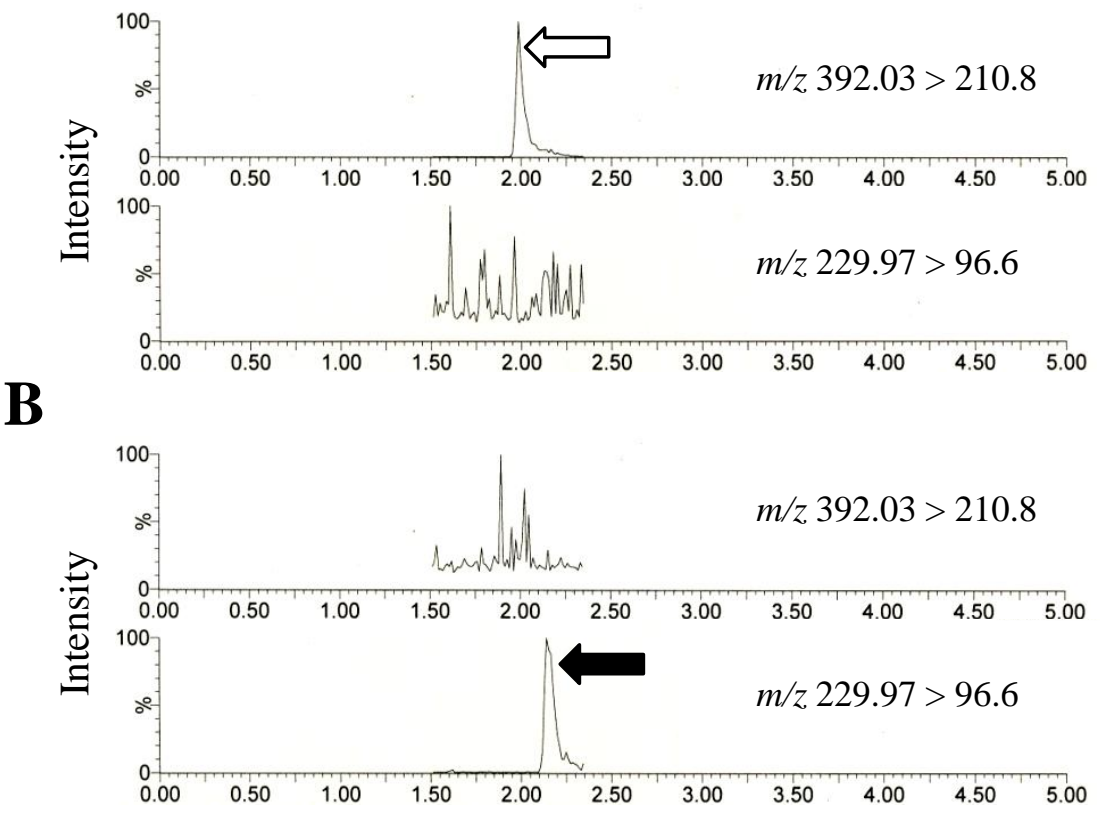

C

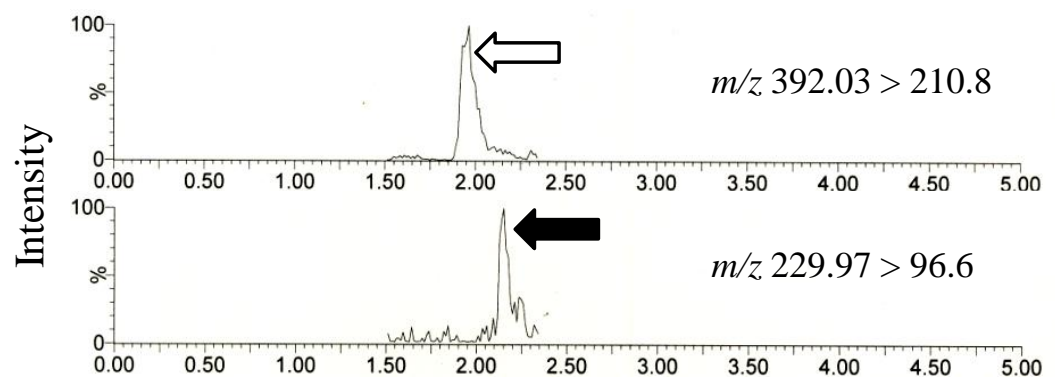

D

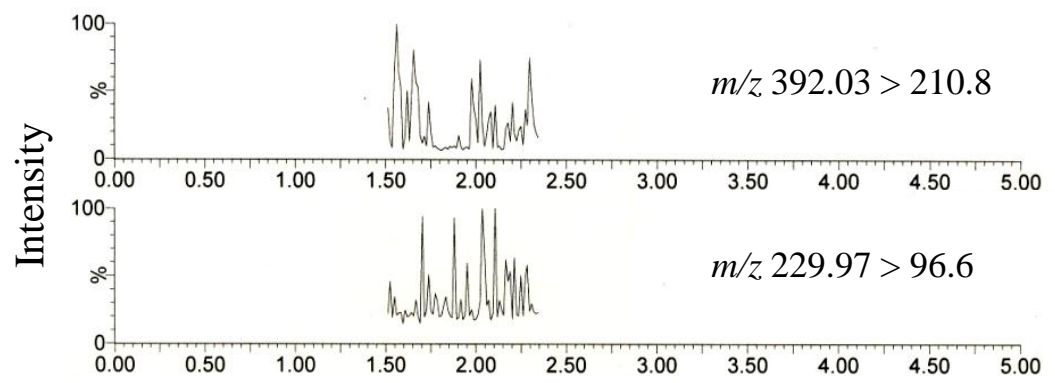

Retention time (min) 

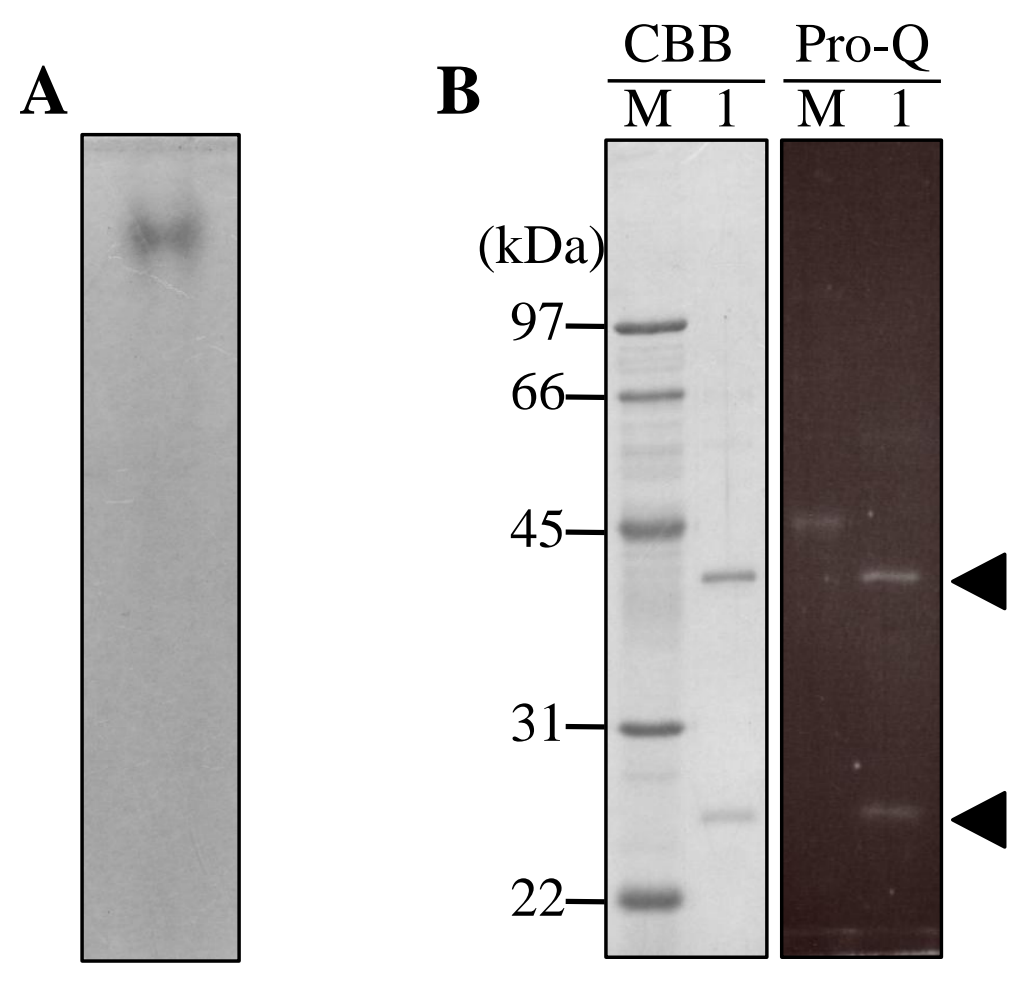

Figure 3. Wakuta et al. 
ATGGCAGCTGCAGGGGAAGTGGTGATGCTTGGTGGCATTCTCCTCCCTCTCCTCCTGGTTGTCGCCGTCTCCGGTGAGCCGCCGCCGATC \begin{tabular}{llllllllllllllllllllllllllllll} 
M & A & A & A & G & E & V & V & M & L & G & G & I & L & L & P & L & L & L & V & V & A & V & S & G & E & P & P & P & I \\
\hline
\end{tabular} AGCCGGAGGAGCTTCCCCGAGGGGTTCATCTTCGGGACGGCCTCGTCGTCGTATCAGTATGAGGGTGGCGCCAGAGAGGGGGGCAGAGGA $\begin{array}{llllllllllllllllllllllllllllll}\mathbf{S} & R & R & S & F & P & E & G & F & I & F & G & T & A & S & S & S & Y & Q & Y & E & G & G & A & R & E & G & G & R & G\end{array}$ CCAAGCATCTGGGACACATTCACACACCAgCACCCAGATAAGATTgCTGACAAAAGCAATGgGgACGTGGCTGCAGACTCCTACCATCTA $\begin{array}{lllllllllllllllllllllllllllllllll}\mathbf{P} & \mathrm{S} & \text { I } & \text { W } & \text { D } & \text { T } & \text { F } & \text { T } & \text { H } & \text { Q } & \text { H } & \text { P } & \text { D } & \text { K } & \text { I } & \text { A } & \text { D } & \text { K } & \text { S } & \text { N } & \text { G } & \text { D } & \text { V } & \text { A } & \text { A } & \text { D } & \text { S } & \text { Y } & \text { H } & \text { L }\end{array}$

TACAAGGAAGATGTGCGCATCATGAAGGATATGGGAGTGGATGCATATAGGTTCTCCATCTCATGGACAAGAATTCTTCCAAATGGAAGT

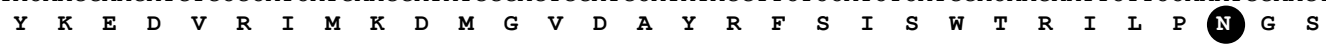

CTGAGCGGTGGAATCAACAGAGAAGGCATCAGCTACTACAACAATTTGATCAATGAACTATTACTGAAAGGGGTGCAACCATTTGTTACC $\begin{array}{llllllllllllllllllllllllllllllll}\text { L } & \text { S } & \text { G } & \text { G } & \text { I } & \text { N } & \text { R } & \text { E } & \text { G } & \text { I } & \text { S } & \text { Y } & \text { Y } & \text { N } & \text { N } & \text { L } & \text { I } & \text { N } & \text { E } & \text { L } & \text { L } & \text { L } & \text { K } & \text { G } & \text { V } & \text { Q } & \text { P } & \text { F } & \text { V } & \text { T }\end{array}$ CTTTTCCACTGGGACTCGCCACAGGCATTAGAAGATAAATATAATGGATTTCTTAGCCCTAATATCATAAATGACTATAAGGAGTACGCT $\begin{array}{lllllllllllllllllllllllllllllllll}\text { L } & \text { F } & \text { H } & \text { W } & \text { D } & \text { S } & \text { P } & \text { Q } & \text { A } & \text { L } & \text { E } & \text { D } & \text { K } & \text { Y } & \text { N } & \text { G } & \text { F } & \text { L } & \text { S } & \text { P } & \text { N } & \text { I } & \text { I } & \text { N } & \text { D } & \text { Y } & \text { K } & \text { E } & \text { Y } & \text { A }\end{array}$ GAAACCTGCTTCAAAGAGTTTGGTGACAGAGTGAAACATTGGATCACCTTCAATGAGCCTTTGAGCTTCTGTGTTGCGGGATATGCATCA $\begin{array}{lllllllllllllllllllllllllllllllll}\text { E } & \text { T } & \text { C } & \text { F } & \text { K } & \text { E } & \text { F } & \text { G } & \text { D } & \text { R } & \text { V } & \text { K } & \text { H } & \text { W } & \text { I } & \text { T } & \text { F } & \text { N } & \text { E } & \text { P } & \text { L } & \text { S } & \text { F } & \text { C } & \text { V } & \text { A } & G & \text { G } & \text { A } & \text { S }\end{array}$ 年

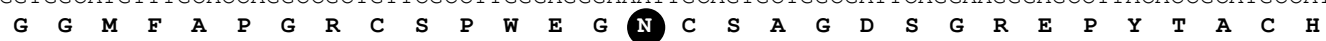

CATCAACTACTTGCTCATGCGGAAACTGTTCGGTTGTACAAAGAGAAATATCAGGTCTTACAAAAGGGGAAGATTGGAATAACTTTGGTC $\begin{array}{llllllllllllllllllllllllllllll}\text { H } & \text { Q } & \text { L } & \text { L } & \text { A } & \text { H } & \text { A } & \text { E } & \text { T } & \text { V } & \text { R } & \text { L } & \text { Y } & \text { K } & \text { E } & \text { K } & \text { Y } & \text { Q } & \text { V } & \text { L } & \text { Q } & \text { K } & \text { G } & \text { K } & \text { I } & \text { G } & \text { I } & \text { T } & \text { L } & \text { V }\end{array}$ TCGAACTGGTTTGTTCCCTTCTCCCGCTCCAAATCCAACATCGATGCTGCAAGGCGTGCTTTAGACTTCATGCTTGGATGGTTTATGGAT

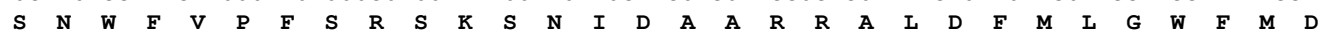
CCCCTAATTAGAGGCGAGTACCCCCTAAGCATGAGAGAATTGGTTCGGAATCGCTTGCCTCAGTTCACTAAAGAACAATCTGAGTTGATC $\begin{array}{lllllllllllllllllllllllllllllll}\mathbf{P} & \text { L } & \text { I } & \text { R } & \text { G } & \text { E } & \text { Y } & \text { P } & \text { L } & \text { S } & \text { M } & \text { R } & \text { E } & \text { L } & \text { V } & \text { R } & \text { N } & \text { R } & \text { L } & \text { P } & \text { Q } & \text { F } & \text { T } & \text { K } & \text { E } & \text { Q } & \text { S } & \text { E } & \text { L } & \text { I }\end{array}$ AAGGGTTCATTCGATTTTATTGGACTTAATTACTACACTTCAAATTATGCTGGTAGCCTTCCTCCATCAAATGGCCTCAATAACAGCTAT

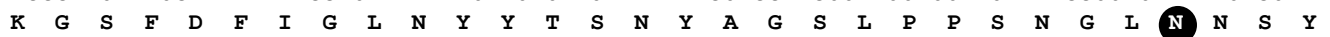
AgtACTGATGCTCGAGCTAATCTTACTGCTGTTCGAAACGGCATTCCCATAGGTCCTCAGGCTGCTTCGCCTTGGCTTTACATCTATCCT $\begin{array}{lllllllllllllllllllllllllllllllll}\mathbf{S} & \mathbf{T} & \mathbf{D} & \mathbf{A} & \mathbf{R} & \mathbf{A} & \mathbf{N} & \mathbf{L} & \mathbf{T} & \mathbf{A} & \mathbf{V} & \mathbf{R} & \mathbf{N} & \mathbf{G} & \mathbf{I} & \mathbf{P} & \mathbf{I} & \mathbf{G} & \mathbf{P} & \mathbf{Q} & \mathbf{A} & \mathbf{A} & \mathbf{S} & \mathbf{P} & \mathbf{W} & \mathbf{L} & \mathbf{Y} & \mathbf{I} & \mathbf{Y} & \mathbf{P}\end{array}$ CAAGGGTTCCGTGAATTGGTGCTTTATGTTAAGGAAAACTATGGCAATCCTACCATCTACATCACCGAAAATGGTGTTGATGAATTCAAC $\begin{array}{lllllllllllllllllllllllllllllllll}Q & G & F & R & E & \text { L } & \text { V } & \text { L } & \text { Y } & \text { V } & \text { K } & \text { E } & \text { N } & \text { Y } & \text { G } & \text { N } & \text { P } & \text { T } & \text { I } & \text { Y } & \text { I } & \text { T } & \text { E } & \text { N } & \text { G } & \text { V } & \text { D } & \text { E } & \text { F } & \text { N }\end{array}$

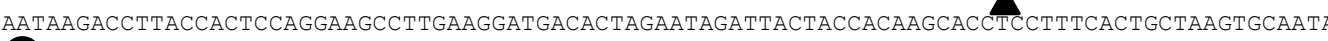
$\begin{array}{lllllllllllllllllllllllllllllllll}\text { N } & \text { K } & \text { T } & \text { L } & \text { P } & \text { L } & \text { Q } & \text { E } & \text { A } & \text { L } & \text { K } & \text { D } & \text { D } & \text { T } & \text { R } & \text { I } & \text { D } & \text { Y } & \text { Y } & \text { H } & \text { K } & \text { H } & \text { L } & \text { L } & \text { S } & \text { L } & \text { L } & \text { S } & \text { A } & \text { I }\end{array}$ AGGGACGGAGCAAATGTGAAGGGATACTTTGCATGGTCGCTGCTTGATAACTTCGAGTGGTCGAACGGCTATACTGTTCGCTTTGGGATA $\begin{array}{lllllllllllllllllllllllllllllll}\mathbf{R} & \mathbf{D} & \mathbf{G} & \mathbf{A} & \mathbf{N} & \mathbf{V} & \mathbf{K} & \mathbf{G} & \mathbf{Y} & \mathbf{F} & \mathbf{A} & \mathbf{W} & \mathbf{S} & \mathrm{L} & \mathrm{L} & \mathbf{D} & \mathbf{N} & \mathbf{F} & \mathbf{E} & \mathbf{W} & \mathbf{S} & \mathbf{N} & \mathbf{G} & \mathbf{Y} & \mathbf{T} & \mathrm{V} & \mathbf{R} & \mathbf{F} & \mathbf{G} & \mathbf{I}\end{array}$

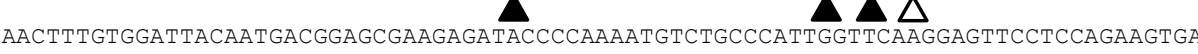

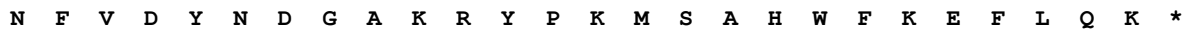


Raucaffricine-O- $\beta$-glucosidase (Rauvolfia serpentina) Furostanol glycoside 26-O- $\beta$-glucosidase (Costus speciosus)

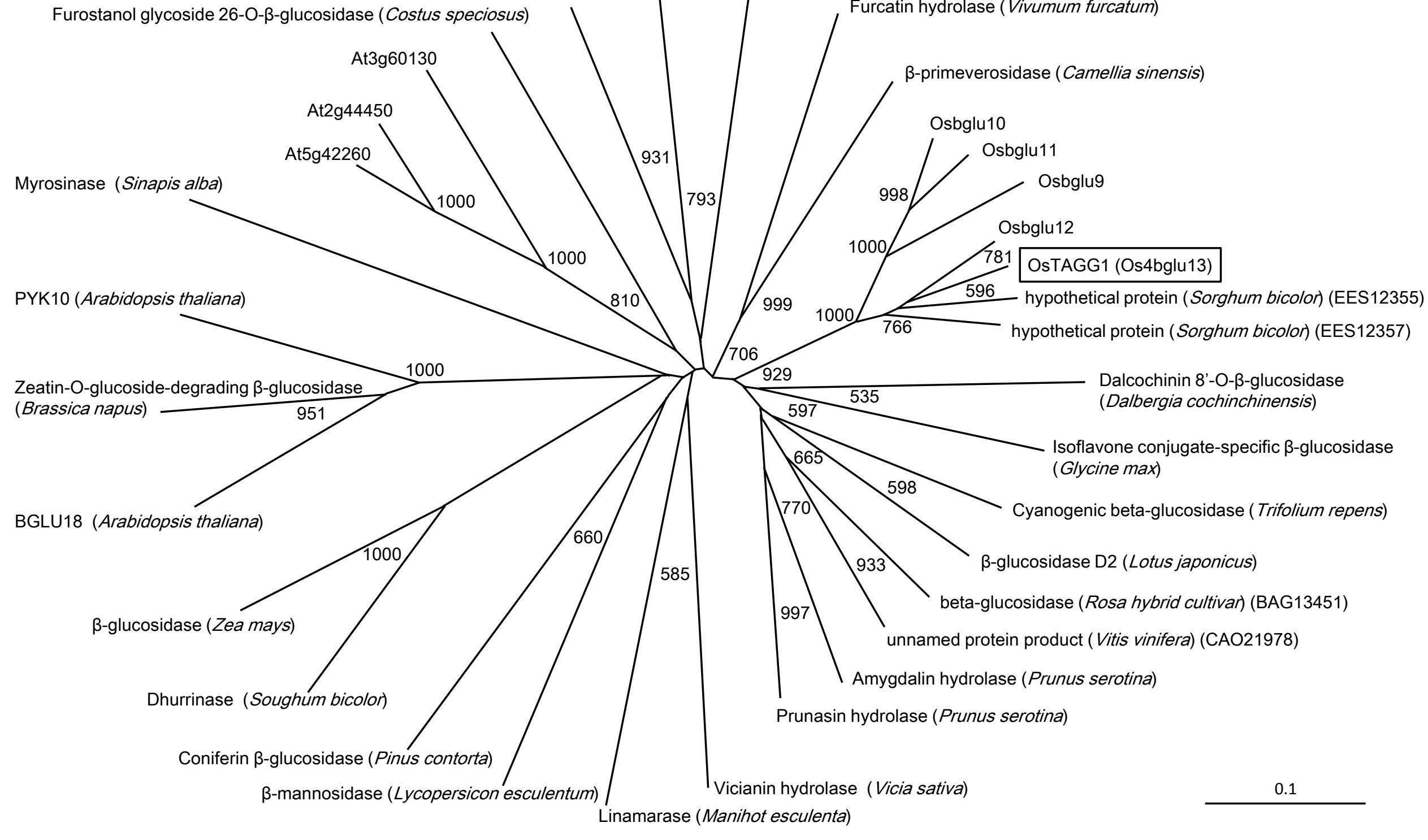

Figure 5. Wakuta et al. 
Table 1

Summary of the purification of OsTAGG1 from rice

\begin{tabular}{llllrr}
\hline Purification step & $\begin{array}{l}\text { Total activity } \\
\text { (units) }\end{array}$ & $\begin{array}{l}\text { Protein } \\
(\mathrm{mg})\end{array}$ & $\begin{array}{l}\text { Specific activity } \\
(\mu \mathrm{mol} / \mathrm{min} / \mathrm{mg})\end{array}$ & $\begin{array}{l}\text { Yield } \\
(\%)\end{array}$ & $\begin{array}{l}\text { Purification } \\
(-\mathrm{fold})\end{array}$ \\
\hline Crude extract & 22 & 25,000 & 0.00088 & 100 & 1 \\
$\left(\mathrm{NH}_{4}\right)_{2} \mathrm{SO}_{4}$ fractionation & 18 & 3,100 & 0.0058 & 82 & 7 \\
$\mathrm{CM}_{-}$Sepharose & 7.6 & 290 & 0.026 & 35 & 30 \\
Con A-Sepharose & 3.0 & 17 & 0.18 & 14 & 200 \\
Superdex 200 & 2.6 & 2.4 & 1.1 & 2.5 & 1,200 \\
Resource S & 0.54 & 0.23 & 2.3 & 1.1 & 4,300 \\
Hydroxyapatite & 0.24 & 0.065 & 3.7 & &
\end{tabular}




\section{Table 2}

Substrate specificity

Substrate

Tuberonic acid glucoside 100

Methyl tuberonic acid glucoside 80

Jasmonoyl-1- $\beta$-glucoside

Salicylic acid glucoside 25

Zeatin glucoside

Linamarin 0

pNP- $\beta$-D-glucoside

178

pNP- $\beta$-D-mannoside

pNP- $\beta$-D-galactoside

pNP- $\beta$-D-cellobioside

1.0

pNP- $\alpha-D$-glucoside

0.45 
Supplementary figure 1
Click here to download S

Click here to download Supplementary Information: suppl. fig.1.pptx 
Supplementary figure 2
Click here to download Supplementary Information: suppl. fig.2.pptx

Supplementary figure 2
Click here to download Supplementary Information: suppl. fig.2.pptx 
Identification of a $\beta$-glucosidase hydrolyzing tuberonic acid glucoside in rice (Oryza sativa $\mathbf{L}$.

Shinji Wakuta ${ }^{\mathrm{a}}$, Shigeki Hamada ${ }^{\mathrm{a}, \mathrm{c} *}$, Hiroyuki Ito ${ }^{\mathrm{b}}$, Hideyuki Matsuura ${ }^{\mathrm{a}}$, Kensuke Nabeta $^{\mathrm{a}}$, and Hirokazu Matsui ${ }^{\mathrm{a}}$

${ }^{a}$ Division of Applied Bioscience, Research Faculty of Agriculture, Hokkaido University, Sapporo 060-8589, Japan

${ }^{\mathrm{b}}$ Department of Materials Engineering, Akita National College of Technology, Akita 011-8511, Japan

${ }^{\mathrm{c}}$ National Institute of Crop Science, National Agriculture and Food Research Organization, Tsukuba, Ibaraki 305-8518, Japan

Rice tuberonic acid glucoside-hydrolyzing $\beta$-glucosidase (OsTAGG1) was purified and examined its property. OsTAGG1 is a glycoprotein consisting of two polypeptides produced by cleavage within a molecule, and showed high specificity for TAG.

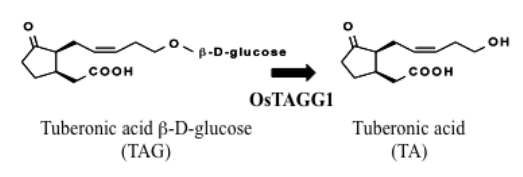

